\title{
Patterns of delayed reinforcement and resistance to extinction'
}

EDWARD L. WIKE, JOHN R. PLATT ${ }^{2}$ AND LINDA PARKER

UNIVERSITY OF KANSAS

\begin{abstract}
An experiment, using rats in a runway, demonstrated that resistance to extinction is affected by the patterns of delayed reinforcement imposed during training.

\section{Problem}

According to cognitive dissonance theory (Schoonard \& Lawrence, 1962) the absolute number of delayed reinforcement trials is a major variable determining resistance to extinction. The purpose of the present experiment was to investigate the effects of three patterns of delayed reinforcement when the absolute number of delayed reinforcements was equated.
\end{abstract}

\section{Method}

The pretraining procedures, including the establishment of a 22-hr. hunger rhythm, handling, and exploration of the runway, extended over 15 days. On the last five days Ss received four rewarded trials daily in which five hurdles, equally spaced in the alley, were gradually increased in size until a 3-in height was achieved. The runway had a 1 -ft start box, 4.5-ft alley, 1.25-ft delay box, and 1.25-ft goal box; all sections were painted black except for the goal box, which was gray。

During acquisition the 26 Sprague-Dawley male albino rats underwent eight trials per day. Group DI $(\mathrm{N}=8)$ received $20-\mathrm{sec}$. delays on all trials on days $1-5$, but had no delays on days 6-10. Group ID $(\mathrm{N}=9)$ was rewarded immediately on days 1-5, but had 20-sec. delay of reinforcement on all trials of days 6-10. Group $R(N=9)$ received four delayed trials and four immediately rewarded trials on days 1-10; a different random sequence of $20-\mathrm{sec}$. delays and non-delays was used each day.

All Ss were extinguished for 20 days. Eight trials were given on day 1 , six trials on day 2 , and four trials per day on the following days. On every extinction trial $S$ was confined for $20 \mathrm{sec}$. to the delay and goal box section of the runway. The 3-in hurdles were present during extinction. Throughout training and extinction the response measure consisted of the time to traverse the middle 3.5-ft section of the alley. The Ss were maintained in the course of the experiment by 1-hr. feedings of small Purina pellets which were presented in the living cages $15 \mathrm{~min}$. after the daily runs. In training two .10-gm pellets of the same type were used as rewards.

\section{Results}

The daily median running times for each $\mathrm{S}$ were calculated. The medians of these medians were converted to logarithms and provided the basis for Fig. 1 and the subsequent analyses of variance. The performance of

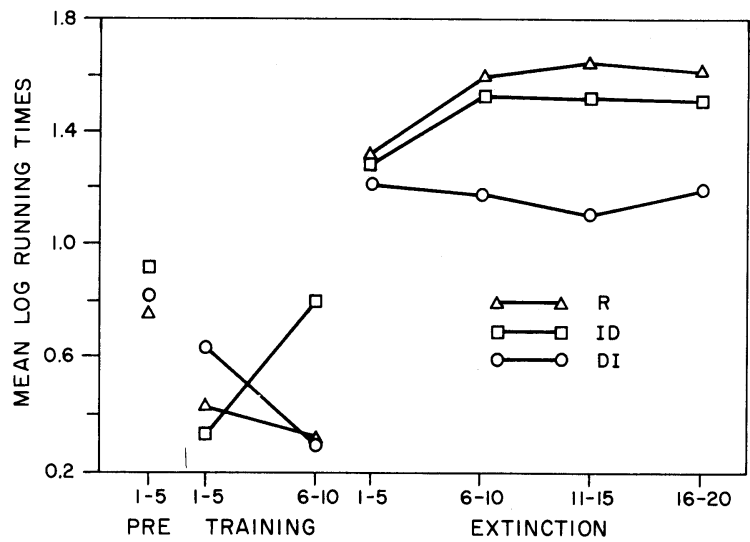

Fig. 1. Mean log running times for the three delay groups for blocks of five days during pretraining (PRE), training, and extinction.

three groups did not differ from chance variation $(F<1)$ in pretraining. The analysis of training disclosed two significant cources of variation: that attributable to Groups $(F=9.16 ;$ df $2 / 23 ; p<.01)$ and to Groups $x$ Trials $(F=47.76 ;$ df $2 / 23 ; p<.001)$. On the average in training, Group $\mathrm{R}$ ran faster $(\mathrm{p}<.01)$ than Groups ID and DI, and DI outperformed $(p<.01)$ ID. The Ss in Group DI displayed a significant decrement $(p<.01)$ in running times when the delays were removed, and Group ID showed a significant increment $(p<.01)$ in running times when delays of reinforcement were instituted. The Ss in Group $\mathrm{R}$ ran faster in the second half of training, but the difference was not statistically significant $(p=.10)$. The analysis of the extinction data revealed three significant sources of variation: groups $(F=5.46$; df $2 / 23 ; p<.05)$, Trials $(\mathrm{F}=10.00 ;$ df $3 / 69 ; \mathrm{p}<.001)$ and Groups $\mathrm{x}$ Trials $(F=4.37$; df $6 / 69 ; p<.01)$. On the average in extinction. DI ran faster $(p<.05)$ than both ID and $R$ which did not differ significantly from each other. Kramer's extension (1956) of Duncan's test was employed for all treatment tests.

Since the groups differed at the end of acquisition, Anderson's shape function technique (1963) was applied to the data. Three $f(n)$ values were obtained for each $S$ using the log median running time for the last half of acquisition as $\mathrm{Ri}$, the log median running time for the last five days in extinction as Roo, and the log medians for the first three blocks of extinction (i.e., days 1-5, 6-10, and 11-15) as $\mathrm{Rn}$ values. An analysis of these $f(n)$ values showed Trials $(F=9.23 ;$ df $2 / 46 ; p<.001)$ and Groups $\mathrm{x}$ Trials ( $\mathrm{F}=4.49 ; \mathrm{df} 4 / 46 ; \mathrm{p}<.01$ ) to be significant. For the first five days of extinction the mean $f(n)$ values of $-.034, .167$, and .342 for groups 
DI, $R$ and ID, respectively, differed significantly $(P=.05)$ from one another. Following these initial days of extinction the $f(n)$ values of the groups converged to a common asymptote.

\section{Discussion}

According to cognitive dissonance theory "...the absolute number of delay trials...should be the major variable in determing resistance to extinction. This should be true even though the animal can predict with complete certainty that the delay will occur on each trial" (Schoonard \& Lawrence, 1962, p. 275). These remarks and others by these authors (e.g.,p.277) suggest that the effects of delayed reinforcement upon resistance to extinction are independent of the patterning of delay. If this implication follows from dissonance theory, then the present data disconfirm the implication. In both analyses of the extinction data differences in extinction performance as a function of delay patterns during acquisition were observed when all three groups had received the same absolute number of delay trials in training. Schoonard \& Lawrence (1962) have also rejected the importance of "uncertainty" of delay in training as a factor in the maintenance of a response in extinction. While we cannot discern the direct relevance of their experiment to this issue, the $\mathbf{R}$ Group in our experiment did not perform well in extinction.

Two additional results of the present investigation are worthy of comment: (1) the superiority in extinction of the DI Group; and (2) the fact that all groups displayed unusual resistance to extinction. With regard to the first finding, it should be noted that Theios (1962) and Jenkins (1962) have reported that $100 \%$ reinforcement given after partial reinforcement in training served to enhance resistance to extinction in comparison with $100 \%$ control groups. Obviously, the patterns of delay em- ployed in the present experiment need to be studied further in the context of partial reinforcement. Three factors may have accounted for the unusual resistance to extinction which was observed in the present study: (1) the change from delays in training to no delays in extinction; (2) the reduction of the number of daily trials in extinction; and/or (3) the presence of hurdles in the alley. To check on the last factor all Ss were given five additional days of extinction and on the last two days the hurdles were removed from the alley. Upon removal of the hurdles, the animals promptly extinguished. Whether or not any other stimulus change would have produced the same result, of course, could not be ascertained.

The present results suggest that more investigations need to be done on patterns of delayed reinforcement. Further studies are also in order in which the effects of confinement and patterns of confinement during extinction are studied.

\section{Referenees}

Anderson, N. H. Comparison of different populations: Resistance to extinction and transfer. Psychol. Rev., 1963, 70, 162-179. Jenkins, H. M. Resistance to extinction when partial reinforcement is followed by regular reinforcement. J. exp. Psychol., 1962, 64 441-450.

Kramer, C. Y. Extension of multiple range tests to group means with unequal numbers of replications. Biometrics, 1956, 12, 307-310.

Schoonard, J., \& Lawrence, D. H. Resistance to extinction as a function of the number of delay of reward trials. Psychol. Rep., 1962, 11, 275-278.

Theios, J. Partial reinforcement effect sustained through blocks of continuous reinforcement. J. exp. Psychol., 1962, 64, 1-6. Notes

1. This research was supported in part by the General Research Fund of the University of Kansas and by an SSTP grant from the NSF.

2. Now at the University of Texas. 